Tarifkonflikt

\section{Wie geht es weiter bei der Bahn?}

Die Tarifbeziehungen der Deutschen Bahn (DB) sind durch zwei Konfliktlagen geprägt, die formalrechtlich unabhängig voneinander sind, inhaltlich-organisatorisch jedoch eng zusammenhängen: der aktuelle Konflikt der Tarifverhandlungen und der langfristige Grundsatzkonflikt um Tarifeinheit versus Tarifpluralität. Der entscheidende $\S 4 \mathrm{a}$ des Tarifeinheitsgesetzes (TEG), der diesen Konflikt lösen soll, lautet: „Soweit sich die Geltungsbereiche nicht inhaltsgleicher Tarifverträge verschiedener Gewerkschaften überschneiden (kollidierende Tarifverträge), sind im Betrieb nur die Rechtsnormen des Tarifvertrags derjenigen Gewerkschaft anwendbar, die zum Zeitpunkt des Abschlusses des zuletzt abgeschlossenen kollidierenden Tarifvertrags im Betrieb die meisten in einem Arbeitsverhältnis stehenden Mitglieder hat."

Das in den meisten Branchen dominierende Prinzip der Industriegewerkschaft (,ein Betrieb, eine Gewerkschaft“) gilt traditionell bei der DB nicht. Die Eisenbahn- und Verkehrsgewerkschaft (EVG) organisiert alle Beschäftigten, die Gewerkschaft Deutscher Lokomotivführer (GDL) vor allem Lokführer:innen und Zugpersonal. Diese Situation ist ungewöhnlich, da Konflikte nicht nur zwischen Arbeitgeberin und Gewerkschaft, sondern auch zwischen den Gewerkschaften bestehen.

Als Teil des Ergebnisses der Schlichtung 2015 bestand ein Grundlagenvertrag zwischen DB und GDL, in dem die DB auf die Anwendung des TEG verzichtete, sodass von beiden Gewerkschaften geschlossene Tarifverträge gelten konnten. Dieser Vertrag sicherte die Unabhängigkeit des Lokführertarifvertrags der GDL und ermöglichte eine konfliktarme Phase der Tarifbeziehungen. Dieser Vertrag lief Ende 2020 aus. Und jetzt insistiert die DB auf der sofortigen Anwendung des TEG (,betriebsbezogenes Mehrheitsprinzip“).

Durch die Entscheidung der DB befürchtet die GDL den Verlust ihrer Verhandlungsmacht bzw. sieht sich sogar in inrer Existenz bedroht. Sie versucht daher, ihre Mitgliederbasis zu stärken, um in möglichst vielen Betrieben Mehrheitsgewerkschaft zu sein. Die GDL hat mehrere Optionen: Werbung von Unorganisierten, was bei den überdurchschnittlichen Organisationsgraden zunehmend schwieriger wird; Abwerbung von Mitgliedern der EVG, was die ohnehin angespannten Beziehungen zwischen

(C) Der/die Autor:in 2021. Open Access: Dieser Artikel wird unter der Creative Commons Namensnennung 4.0 International Lizenz veröffentlicht (creativecommons.org/licenses/by/4.0/deed.de).

Open Access wird durch die ZBW - Leibniz-Informationszentrum Wirtschaft gefördert. beiden Gewerkschaften weiter belastet; Ausdehnung über den Bereich von Lokführer:innen und gesamtem Zugpersonal hinaus auf alle im „System Eisenbahn relevanten Berufsgruppen“ (z. B. Fahrzeug- und Fahrweginstandhaltung). Dieser Ende 2020 angekündigte Schritt intensiviert ebenso den bestehenden Konflikt.

Die Befürwortenden des TEG erwarteten in der politischen Auseinandersetzung weniger Konkurrenz zwischen den beteiligten Gewerkschaften sowie „Anreize für Kooperation und Abstimmung“. Das von den Gegner:innen des TEG befürchtete Gegenteil ist eingetreten: Die Konkurrenz nimmt zu. Nach einer Urabstimmung organisiert die GDL im Spätsommer 2021 mehrere zeitlich befristete, sich verschärfende Streiks im Güter- und Personenverkehr. Der Konflikt lässt sich nicht durch Anwendung der komplizierten, unbestimmten Regelungen des TEG beilegen, sondern nur durch Verhandlungen der Tarifparteien.

Das Ziel besteht in der Sicherung des Grundrechts der Koalitionsfreiheit, deren Voraussetzungen sich durch die zunächst faktische, dann auch rechtliche Ablösung der Tarifeinheit durch Tarifpluralität verändert haben. Erforderlich sind auf beiden Seiten Kompromisse durch explizite Anerkennung dieser veränderten Rahmenbedingungen: Die notwendige Voraussetzung ist entweder die Verlängerung des alten Grundlagentarifvertrags oder der Abschluss eines neuen. Dieser Vertrag kann langfristig gelten und verlängert werden. Die mehrjährigen Erfahrungen zeigen, dass diese Option zur Sicherung von Tarifpluralität realistisch ist.

Für eine neue Rahmenregelung sind folgende Konzessionen notwendig: Die GDL erkennt den Status quo an und verzichtet auf Versuche, ihre Organisationsdomäne auszudehnen, um die Tarifverträge der EVG zu verdrängen. Eine Erweiterung war der GDL Anfang der 2000er Jahre gelungen, als sie neben den Lokführer:innen das gesamte Fahrpersonal für sich reklamierte. Die DB akzeptiert die GDL als unabhängige Tarifpartei durch Anwendungsgarantien ihrer Kollektivverträge, sichert dadurch den "Betriebsfrieden“ und verhindert weitere Arbeitskämpfe. Der „Preis“ der DB sind weiterhin höhere Transaktionskosten (bei den Verhandlungen und bei der Umsetzung von Kollektivverträgen) als bei Tarifeinheit.

Diese Konzessionen sind für beide Seiten weitreichend und bedeuten Sprünge über den eigenen Schatten. Ihre einzige Alternative ist allerdings die Perpetuierung des schwelenden Grundlagenkonflikts, woran keiner der Beteiligten Interesse haben kann. 